\title{
What Will It Take? The Quest to Achieve Indexing by the National Library of Medicine
}

\author{
Mary H H Ensom
}

$\mathrm{T}$

become indexed in MEDLINE has been a goal of the

Canadian Journal of Hospital Pharmacy (CJHP) for as long as those of us on the Editorial Board can remember. We believe that the benefits of achieving indexing in MEDLINE are enormous. First, indexing will provide more exposure for the work of authors who publish in the CJHP. That is, authors' articles will be more widely disseminated and more readily available to other clinicians and scientists around the world, who can then incorporate this new knowledge into their practice or build on the published work. Second, indexing will lead to more high-quality submissions. I have heard from more than a few of my academic colleagues who work in a "publish or perish" environment that they do not submit papers to the CJHP because "it is not indexed". Once we achieve indexing status, however, authors will receive more recognition, more citations, and higher impact for their published papers and therefore will be more likely to submit their high-quality work to CJHP. Third, indexing is expected to improve the value of the Journal not only for authors but also for readers, subscribers, advertisers, and its owner, the Canadian Society of Hospital Pharmacists (CSHP), for all the reasons described above.

If you have searched PubMed recently, you may have noticed that you are already able to retrieve citations of articles published in the CJHP. This is because the Journal was successful in its 2010 application for archiving in PubMed Central (PMC), the US National Institutes of Health's free digital archive of biomedical and life sciences journal literature (www.ncbi.nlm.nih.gov/pmc/). PMC is part of the larger PubMed service, which also includes MEDLINE, the primary international online bibliographic citation database of biomedical and life sciences journal literature. PubMed now includes a link to the material that $C J H P$ has available on the PMC site.

This is great news, but PMC archiving is only one step toward indexing. That is, at the PubMed site, CJHP citations are denoted as "PubMed - in process" or "PubMed". Only after the CJHP is indexed by the National Library of Medicine (NLM) will it say "PubMed - indexed for MEDLINE".

Currently, about 5000 journal titles are indexed in MEDLINE. The Director of the NLM decides whether or not to index a journal, on the basis of considerations of both scientific quality and policy. Specifically, the NLM's Literature Selection Technical Review Committee (LSTRC) reviews and assesses the quality of journals, while NLM's Board of Regents determines the mix of types of journals to be indexed, according to considerations of program relevance.'

In fact, our Journal was indexed in MEDLINE over the period 1975 to 1997 . While the specific reasons for halting our indexing are unknown, the NLM has been known to drop currently indexed journals to accommodate new ones.

In 2004, the CJHP submitted an application for indexing that was unsuccessful. Although we would have been allowed to reapply after a minimum of 3 years, the Editorial Board believed it best to hold off until we were more confident of success. As such, we have been "beefing up" the Journal, ${ }^{2}$ while carefully examining the NLM's criteria for indexing and scrutinizing what it took for other journals to achieve indexing status.

I would like to take this opportunity to describe some of the additional changes we are planning for the CJHP and to explain why we are advocating these changes and why we believe we can be successful in our effort to achieve indexing status.

In planning for our next application for indexing (in early 2015), we have adopted the following primary goals: (1) to improve the CJHP's scope and coverage, making the Journal more internationally relevant; (2) to continue improving the quality of the CJHP's review articles, clinical research papers, case reports, and editorials; (3) to further improve the LSTRC's perception of the strength and credibility of the CJHP's Editorial Board and the Journal's editorial independence; (4) to 
improve the $C J H P$ 's perceived importance to critical audiences, specifically clinical pharmacists, educators and preceptors, hospital pharmacy administrators, and researchers; (5) to ensure that the CJHP's content meets the LSTRC criteria for indexing; and (6) to heed the NLM's caveat that "publications that consist primarily of reprinted articles, reports of association activities, abstracts of the literature, news items or book reviews, will not usually be indexed".

Specifically, we plan the following changes, among others. We will have at least one individual from outside North America on our Editorial Board at all times. We will regularly solicit guest editorials from prominent pharmacists (including recipients of the CSHP Distinguished Service Award and individuals based outside of Canada). Starting with the current issue, we have updated our column categories as follows: Editorial, Original Research, Reviews, Clinical Practice, Pharmacy Education, Social and Administrative Pharmacy, Case Reports, Books and Other Media, Letters, and the ever-popular Point Counterpoint; the Practice Spotlight column has now been eliminated, effective with this issue. We have started formally soliciting systematic reviews from experts across Canada and, in conjunction with the CSHP Research Committee, will be identifying topics and prospective authors for a Research Primer series. These are just a few of the changes that our readers will see over the coming months.

For now, we will continue publishing reports from the CSHP Presidential Team and Executive Director, as well as abstracts from the Society's Professional Practice Conference and Summer Educational Session. We are in discussions with the CSHP Executive Committee and Council about the best way to reduce the "less scientific" content (i.e., Society news-like items) to follow suit with other successfully indexed journals.
Only $20 \%$ to $25 \%$ of journal titles reviewed by the NLM are selected for indexing. ${ }^{3}$ However, if we do what it takes, the CJHP will reach this goal!

\section{References}

1. Fact sheet MEDLINE® journal selection. Bethesda (MD): National Institutes of Health (US), National Library of Medicine; 2012 [cited 2012 Sep 17]. Available from: www.nlm.nih.gov/pubs/factsheets/jsel.html

2. Ensom MHH. Facts and comparisons: journal updates and performance indicators [editorial]. Can J Hosp Pharm. 2010;63(3):281-3.

3. FAQ: journal selection for MEDLINE® indexing at NLM. Bethesda (MD): National Institutes of Health (US), National Library of Medicine; 2012 [cited 2012 Sep 17]. Available from: www.nlm.nih.gov/pubs/factsheets/j_sel_faq.html

Mary H H Ensom, PharmD, FASHP, FCCP, FCSHP, FCAHS, is Professor, Faculty of Pharmaceutical Sciences, and Distinguished University Scholar, The University of British Columbia, and Clinical Pharmacy Specialist, Children's \& Women's Health Centre of British Columbia, Vancouver, British Columbia. She is also the Editor of the CJHP.

Competing interests: None declared.

\section{Address correspondence to:}

Dr Mary H H Ensom

Department of Pharmacy (OB7)

Children's \& Women's Health Centre of British Columbia

4500 Oak Street

Vancouver BC V6H 3N1

e-mail: ensom@mail.ubc.ca

\section{Acknowledgements}

I would like express my sincerest appreciation to all of the Associate Editors for their invaluable contributions and especially to Jim Tisdale for taking the lead in drafting a strategic plan for achieving indexing of the CJHP by the NLM.

\section{ON THE FRONT COVER}

\section{Troll Falls Kananaskis Country, Alberta}

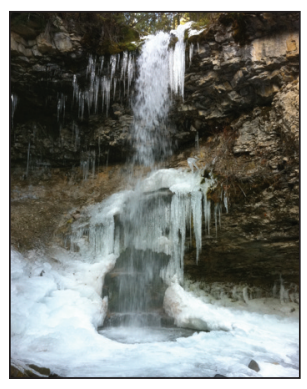

This photograph was taken in late October 2011, during a hike in Kanaskis Country, a beautiful park area within Alberta's Rocky Mountain range. Along with many other trails, the Troll Falls trail begins not far from the Kananaskis Village Centre, about an hour's drive from Calgary, Alberta. CSHP member Liz Glashan used her iPhone 4 to take the photo.
The CJHP would be pleased to consider photographs featuring Canadian scenery taken by CSHP members for use on the front cover of the journal. If you would like to submit a photograph, please send an electronic copy (minimum resolution $300 \mathrm{dpi}$ ) to Colleen Drake at cdrake@cshp.ca. 